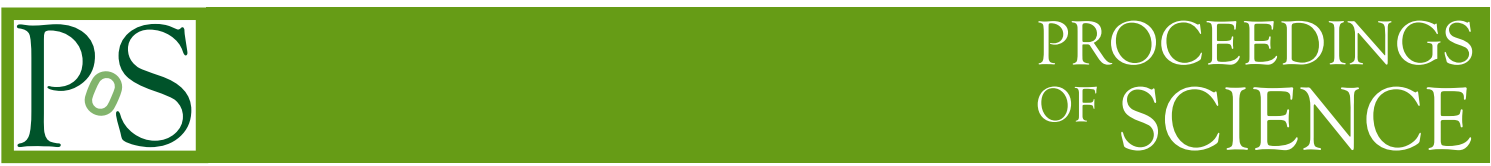

\title{
Gamma-gamma absorption in $\gamma$-ray binary systems
}

\author{
DC du Plooy* and B van Soelen \\ Department of Physics, University of the Free State, PO Box 339, Bloemfontein 9300, South \\ Africa \\ E-mail: duplooydcaufs.ac.za
}

\begin{abstract}
Gamma-ray binaries are a subclass of high-mass binary systems and are generally distinguished by their spectral energy distributions peaking above $1 \mathrm{MeV}$. Gamma-ray binaries consist of an $\mathrm{O}$ or $\mathrm{B}$ type star and an orbiting compact object which is either a neutron star or a black hole. The nature of the compact object in $\gamma$-ray binaries remains unknown except for two systems, namely PSR B1259-63 and PSR J2032+4127, where the compact objects have been identified as pulsars. In the case of a neutron star compact object, the very high energy (VHE) emission mechanism is believed to originate from the interaction between the stellar and pulsar winds. Generally, $\gamma$-ray binary light-curves show that the maximum in the $\mathrm{GeV}$ and $\mathrm{TeV}$ emission occurs at different orbital phases, with the maximum $\mathrm{GeV}$ emission occurring at superior conjunction and the maximum $\mathrm{TeV}$ emission occurring at inferior conjunction. Therefore, $\gamma \gamma$ absorption might prove useful in constraining the $\mathrm{TeV}$ emission region by studying how it affects the observed spectrum. We have calculated the $\gamma \gamma$ absorption in six of the seven known $\gamma$-ray binaries and the results are presented in this paper.
\end{abstract}

7th Annual Conference on High Energy Astrophysics in Southern Africa - HEASA2019

28 - 30 August 2019

Swakopmund, Namibia

*Speaker. 


\section{Introduction}

Gamma-ray binaries are a subclass of high-mass binary systems and consist of a compact object, either a neutron star or a black hole, in orbit around an O or B type star. Gamma-ray binaries show emission up to $\mathrm{TeV}$ energies, produced by the inverse Compton scattering of electrons off stellar photons. Generally in these systems the nature of the compact object remains unknown, except for two sources, namely PSR B1259-63 and PSR J2032+4127, where the compact objects have been identified as pulsars $[1,2,3]$. In the case of a neutron star compact object, the neutron star is most likely a young pulsar which is spinning too rapidly for accretion to occur. Accretion of material onto the poles of a neutron star would short out the electric field responsible for pulsed radio emission, which contradicts the detections made in the two aforementioned $\gamma$-ray binary systems. However, the possibility of a microquasar scenario is still open to debate for the other systems, in which case the $\gamma$-rays would be produced via Compton scattering close to the base of the relativistic jet $[4,5]$. In the case of a pulsar compact object, the winds originating from the pulsar and companion star produce a shock situated between the two orbiting bodies and due to the stellar wind domination over the pulsar wind, the shock structure takes the shape of a cometary tail flowing over the pulsar. At the apex of the shock high energy particles are accelerated to VHE similarly to pulsar wind nebulae as described by e.g. Gaensler \& Slane [6]. However, due to the dense photon field the resulting $\gamma \gamma$ absorption will also be high [7]. A second possible location for the emission has been discussed, situated further along the cometary tail, at the Coriolis turnover $[8,9,10]$. In this paper we include results on measuring the level of $\gamma \gamma$ absorption in $\gamma$-ray binaries by constructing absorption maps. These absorption maps provide valuable insight that will be used to place constraints on where the observed VHE emission can originate from.

\section{Theory}

Gamma-ray photons will undergo $\gamma \gamma$ absorption in the photon field of the star when

$$
E_{\gamma} \epsilon \geq \frac{2 m_{e}^{2} c^{4}}{\left(1-\overrightarrow{e_{\gamma}} \cdot \overrightarrow{e_{\star}}\right)}
$$

where $E_{\gamma}$ is the energy of the $\gamma$-ray, $\epsilon$ is the energy of the optical target photon, $m_{e}$ is the electron mass, $c$ is the speed of light, and $\overrightarrow{e_{\gamma}}$ and $\overrightarrow{e_{\star}}$ are the direction of motion of the $\gamma$-ray and optical photon respectively. Following the description in Dubus [7], the opacity of a $\gamma$-ray photon produced with energy in the binary system, travelling in a direction $\overrightarrow{e_{\gamma}}$, can be described by a quadruple integral which is dependent on the path length $\ell$, the solid angle over the star $d \Omega=d \mu d \phi$ (where $\mu=\cos \theta$; refer to Fig. 1), and the energy of the target photon, $\epsilon$, and can be written as

$$
\tau_{\gamma \gamma}=\int_{0}^{\infty} \int_{\mu_{\min }}^{1} \int_{0}^{2 \pi} \int_{\epsilon_{\min }}^{\epsilon_{\max }} n_{\epsilon} \sigma_{\gamma \gamma}\left(1-\overrightarrow{e_{\gamma}} \cdot \overrightarrow{e_{\star}}\right) d \epsilon d \phi d \mu d \ell
$$

where $n_{\epsilon}$ is the stellar photon number density, $\sigma_{\gamma \gamma}$ is the interaction cross-section, $\mu_{\min }=$ $\sqrt{\left(1-\left(R_{\star} / d\right)^{2}\right)}, d$ is the distance to the centre of the star. The $\gamma \gamma$ cross-section is given 


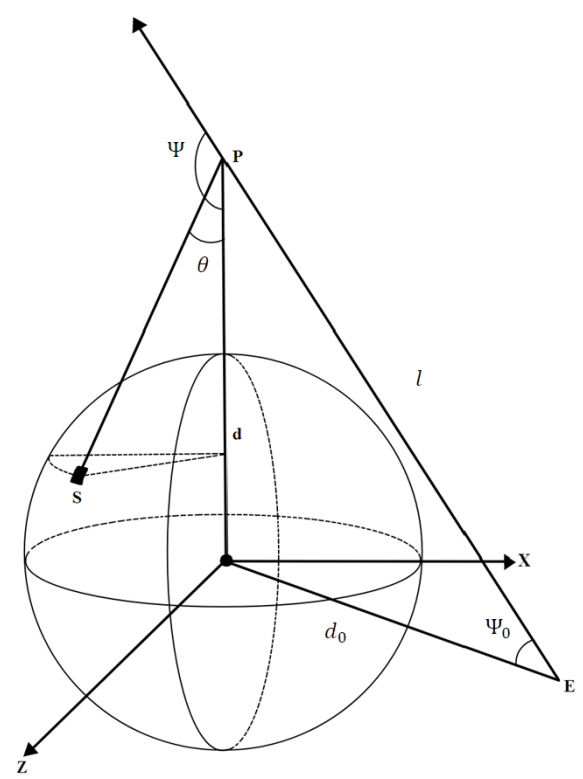

Figure 1: The geometry of the $\gamma$-ray and stellar photon interaction where the sphere represents the optical companion. The emission region of the $\gamma$-ray photon is given by $\mathrm{E}$, a distance $d_{0}$ from the centre of the star. The $\gamma$-ray photon travels a distance $\ell$ before it interacts with the stellar photon, which is produced at $S$. The angles $\Psi_{0}, \Psi$ and $\theta$ represent the $\gamma$-ray emission angle, the interaction angle, and the co-latitude respectively.

by [11]

$$
\sigma_{\gamma \gamma}=\frac{3}{16} \sigma_{\mathrm{T}}\left(1-\beta^{2}\right)\left[\left(3-\beta^{4}\right) \ln \left(\frac{1+\beta}{1-\beta}\right)-2 \beta\left(2-\beta^{2}\right)\right],
$$

where $\sigma_{\mathrm{T}}$ is the Thomson cross-section and $\beta$ is a unit-less parameter given by

$$
\beta=\sqrt{1-\frac{2 m_{e}^{2} c^{4}}{\epsilon E_{\gamma}\left(1-\overrightarrow{e_{\gamma}} \cdot \overrightarrow{e_{\star}}\right)}} .
$$

\section{Results}

Maps of the level of $\gamma \gamma$ absorption for $E_{\gamma}=1 \mathrm{TeV}$ are shown in Fig. 2 with the observer positioned at the bottom of the figure. The colour gradient shows the level of absorption. These maps were constructed by determining the absorption, using equation 2.2, and constrained to within a multiplication of five times the original orbit size. The binary parameters are listed in Table 1.

\section{Discussion \& Conclusions}

Generally, because of the high angular dependence of inverse Compton scattering what is expected is that the maximum $\mathrm{GeV}$ and $\mathrm{TeV}$ emission occurs near or at superior 
Table 1: Binary parameters used in the model. The parameters are described as follows; $P_{\text {orb }}$ is the orbital period, $e$ is the eccentricity, $\omega$ is the argument of periastron, $i$ is the orbital inclination, $T_{\star}$ is the effective stellar surface temperature, $R_{\star}$ is the stellar radius, $M_{\star}$ is the companion stellar mass, and $M_{\text {compact }}$ is the compact object mass. Where values are constrained by observations the error is also reported in brackets.

\begin{tabular}{|c|c|c|c|c|c|c|}
\hline & $\begin{array}{c}\text { LS } \\
5039 \bowtie\end{array}$ & $\begin{array}{c}\text { PSR } \\
\text { B1259-63 }^{+}\end{array}$ & $\begin{array}{c}\text { LS I } \\
61^{\circ} 303^{\star}\end{array}$ & $\begin{array}{c}1 \text { FGL } \\
\text { J1018.6-5856 * }\end{array}$ & $\begin{array}{c}\text { HESS } \\
\text { J0632+057 }\end{array}$ & LMC P3 $^{\diamond}$ \\
\hline$P_{\text {orb }}$ (days) & $3.90603(8)$ & $1236.724526(6)$ & $26.496(3)$ & $16.544(8)$ & $315(5)$ & 10.301(2) \\
\hline$e$ & $0.24(8)$ & $0.86987970(6)$ & $0.54(3)$ & 0.31 & $0.83(8)$ & $0.40(7)$ \\
\hline$\omega\left(^{\circ}\right)$ & $212(5)$ & $138.665013(11)$ & $41(6)$ & $89(30)$ & $129(17)$ & $11(12)$ \\
\hline$i\left({ }^{\circ}\right)$ & 64 & $153.3_{-3.0}^{+3.2}$ & 60 & 64 & 80 & 50 \\
\hline $\mathrm{T}_{\star}(\mathrm{K})$ & 39000 & 33500 & 22500 & 38900 & 30000 & 40000 \\
\hline $\mathrm{R}_{\star}\left(R_{\odot}\right)$ & 9.3 & 9.2 & 10 & 10.1 & 8 & 15 \\
\hline $\mathrm{M}_{\star}\left(M_{\odot}\right)$ & 23 & 29.8 & 12 & 31 & 16 & 42 \\
\hline $\mathrm{M}_{\text {compact }}\left(M_{\odot}\right)$ & 1.4 & 1.4 & 1.4 & 1.4 & 1.4 & 1.4 \\
\hline
\end{tabular}

$\bowtie[12,13,14] ;+[15,16,17] ; \star[13,18] ; *[19,20,21] ; \ddagger[22,23,24] ; \diamond[25,26,27]$

conjunction. However, if the VHE photons exceed the energy threshold for $\gamma \gamma$ absorption this will affect the observed $\mathrm{TeV}$ spectrum. As can be seen in figure 1, the majority of the $\gamma \gamma$ absorption occurs directly behind the companion star, relative to an observer positioned below the figure. This would result in the $\gamma$-ray photons travelling towards the observer to encounter a much denser photon field. Therefore because of the higher amount of $\gamma \gamma$ absorption of $\mathrm{TeV}$ photons at superior conjunction, what is observed is that the maximum $\mathrm{GeV}$ emission occurs at superior conjunction while the maximum $\mathrm{TeV}$ emission occurs at inferior conjunction. This can explain the difference between the $\mathrm{GeV}$ and TeV light-curves, in systems like LS 5039 and LMC P3 [7, 28, 27]. Other than the high angular dependency, $\gamma \gamma$ absorption is also highly dependent on the binary separation. By comparing LS 5039 and HESS J0632+057 one can see that for closely separated binary systems, a significant degree of absorption still occurs at or near inferior conjunction. In $\gamma$-ray binaries, $\gamma \gamma$ absorption will result in a decrease in the observed flux and will harden the $\mathrm{TeV}$ spectrum because the absorption will be highest in the around at few $100 \mathrm{~s}$ of $\mathrm{GeV}$ energies. The $\gamma \gamma$ calculations discussed here will be used to predict how the flux and the photon index will change during the orbit for emission originating at different distances along the bow shock. The main objective of this study is to produce predictions that can constrain the possible locations of the $\mathrm{TeV}$ emission and which can be tested with the upcoming Cherenkov Telescope Array.

\section{Acknowledgments}

The numerical calculations were performed using the University of the Free State High Performance Computing Unit. 

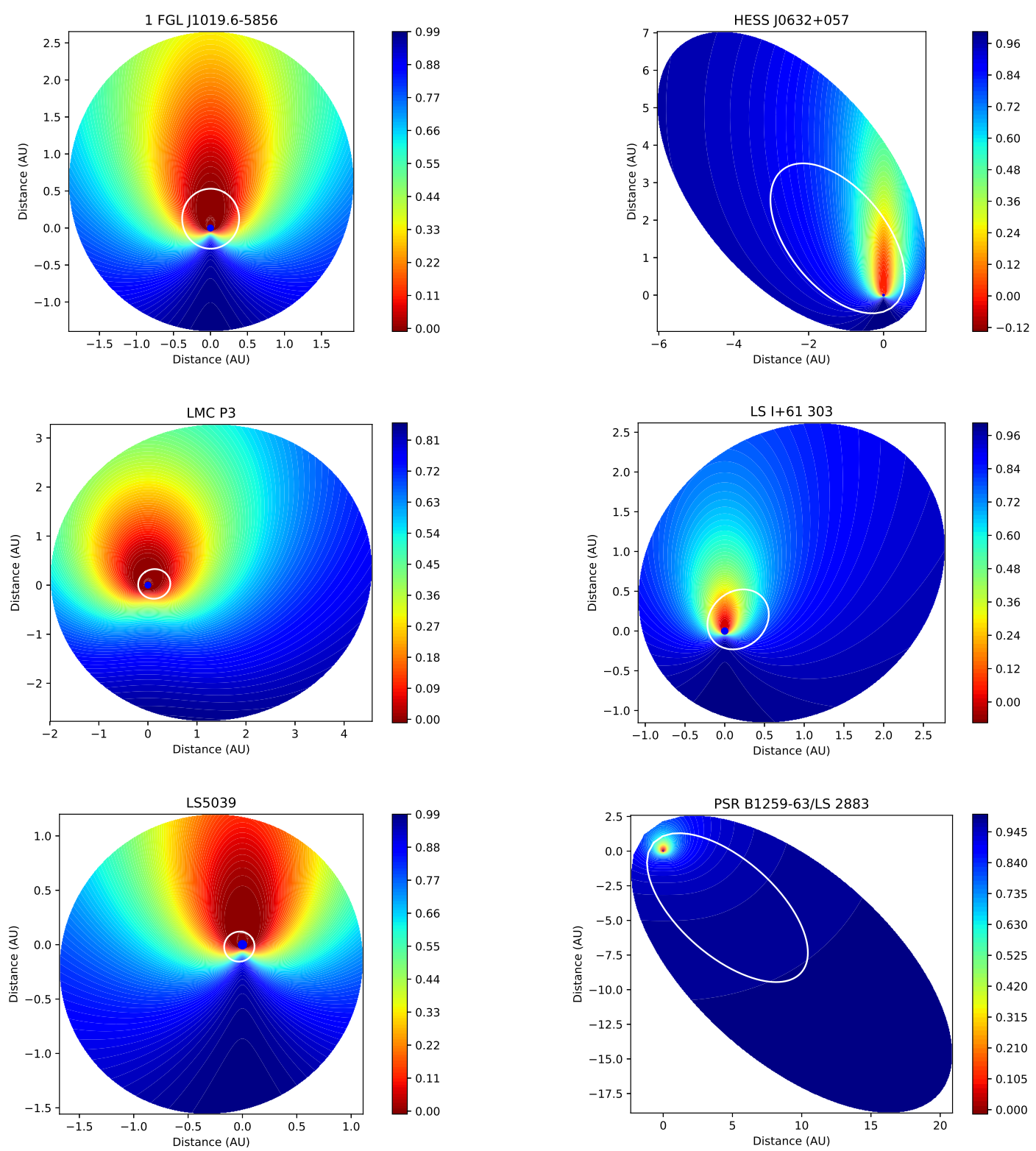

Figure 2: The absorption maps around the $\gamma$-ray binaries for a $1 \mathrm{TeV} \gamma$-ray photon for an observer positioned at the bottom of the page. The orbit of the compact object is given by a solid white line with the companion star to scale. The colour scale depicts the amount of absorption (as $\exp \left(\tau_{\gamma \gamma}\right)$ ).

\section{References}

[1] S. Johnston, R. N. Manchester, A. G. Lyne, et al., PSR 1259-63: A Binary Radio Pulsar with a Be Star Companion AEA 387 (1992) 37

[2] F. Camilo, P. S. Ray, S. M. Ransom, M. Burgay, et al., Radio Detection of LAT PSRs J1741-2054 and J2032+4127: No Longer Just Gamma-ray Pulsars ApJ 705 (2009) 1

[3] A. A. Abdo, M. Ackermann, M. Ajello, W. B. Atwood, et al., Detection of 16 Gamma-Ray Pulsars Through Blind Frequency Searches Using the Fermi LAT Science 325 (2009) 840 
[4] C. D. Dermer, M. Böttcher, Model for High-Energy Radiation from the Black-Hole Microquasar LS 5039 AAS 207 (2005) 9007

[5] F. Jaron, R. Sharma, M. Massi, L. Fuhrmann, et al., Radio QPO in the $\gamma$-ray-loud X-ray binary LS I +61 303 MNRAS 471 (110)

[6] B. M. Gaensler, P. O. Slane, The Evolution and Structure of Pulsar Wind Nebulae A\&A 44 (2006) 17

[7] G. Dubus, Gamma-ray absorption in massive X-ray binaries AEA 451 (2006) 9

[8] V. Bosch-Ramon, M. V. Barkov, Large-scale flow dynamics and radiation in pulsar $\gamma$-ray binaries $A \mathcal{E} A 535$ (2011) A20

[9] V. Bosch-Ramon, M. V. Barkov, D. Khangulyan, M. Perucho, Large-scale flow dynamics and radiation in pulsar $\gamma$-ray binaries A\&A 544 (2012) A59

[10] V. Zabalza, V. Bosch-Ramon, et al., Unraveling the high-energy emission components of gamma-ray binaries A\&A 551 (2013) A17

[11] J. M. Jauch, F. Rohrlich, Texts and Monographs in Physics $2^{\text {nd }} e d$. New York: Springer (1967)

[12] M. Ribó, J. M. Paredes, G. E. Romero, et al., LS 5039: A runaway microquasar ejected from the galactic plane AEA 384 (2002) 954

[13] M. V. McSwain, D. R. Gies, W. Huang, et al., The N Enrichment and Supernova Ejection of the Runaway Microquasar LS 5039 ApJ 600 (2004) 927

[14] G. E. Sarty, T. Szalai, L. L. Kiss, et al., The $\gamma$-ray binary LS 5039: mass and orbit constraints from MOST observations MNRAS 411 (2011) 1293

[15] R. M. Shannon, S. Johnston, R. N. Manchester, The kinematics and orbital dynamics of the PSR B1259-63/LS 2883 system from 23 yr of pulsar timing MNRAS 437 (2014) 3255

[16] J. C. Miller-Jones, A. T. Deller, R. M. Shannon, et al., The geometric distance and binary orbit of PSR B1259-63 MNRAS 479 (2018) 4849

[17] I. Negueruela, M. Ribó, A. Herrero, et al., Astrophysical Parameters of LS 2883 and Implications for the PSR B1259-63 Gamma-ray Binary ApJ 732 (2011) L11

[18] C. Aragona, M. V. McSwain, E. D. Grundstrom, et al., The Orbits of the $\gamma$-Ray Binaries LS I+61 303 and LS 5039 ApJ 689 (2009) 514

[19] H. An, E. Belim, V. Bhalerao, et al., Broadband X-Ray Properties of the Gamma-Ray Binary 1FGL J1018.6-5856 ApJ 806 (2015) 166

[20] I. M. Monageng, V. A., McBride, L. J. Townsend, et al, The Orbit of the Gamma-Ray Binary 1FGL J1018.6-5856 ApJ 847 (2017) 68

[21] V. J. Napoli, M. V. McSwain, M. Viginia, et al., The Distance of the $\gamma$-Ray Binary 1FGL J1018.6-5856 PASP 123 (2011) 1262

[22] C. Aragona, M. V. McSwain, M. Virginia, M. De Becker, HD 259440: The Proposed Optical Counterpart of the r-ray Binary HESS J0632+057 ApJ 724 (2010) 306

[23] J. Casares, M. Ribó, I. Ribas, et al., On the binary nature of the $\gamma$-ray sources AGL J2241+4454 (= MWC 656) and HESS J0632+057 (= MWC 148) MNRAS 421 (2012) 1103

[24] E. Aliu, S. Archambault, S., T. Aune, et al., Long-term TeV and X-Ray Observations of the Gamma-Ray Binary HESS J0632+057 ApJ 780 (2014) 168 
[25] R. H. D. Corbet, L. Chomiuk, M. J. Coe, et al., A Luminous Gamma-ray Binary in the Large Magellanic Cloud ApJ 829 (2016) 105

[26] G. Pietrzyński, D. Graczyk, W. Gieren, et al, An eclipsing-binary distance to the Large Magellanic Cloud accurate to two per cent Nature 495 (2013) 76

[27] B. van Soelen, N. Komin, A. Kniazev, P. Väisänen, The orbital parameters of the gamma-ray binary LMC P3+ MNRAS 484 (2019) 4347

[28] M. Böttcher, C. D. Dermer, Photon-Photon Absorption of Very High Energy Gamma Rays from Microquasars: Application to LS 5039 ApJ 634 (2005) 81

[29] H.E.S.S. Collaboration, et al., Detection of variable VHE gamma-ray emission from the extra-galactic gamma-ray binary LMC P3 AEA 610 (2018) L17 